\title{
Effectiveness of Australian youth suicide prevention initiatives
}

\author{
Andrew Page, Richard Taylor, David Gunnell, Greg Carter, Stephen Morrell and Graham Martin
}

\section{Background}

After an epidemic rise in Australian young male suicide rates over the 1970s to 1990s, the period following the implementation of the original National Youth Suicide Prevention Strategy (NYSPS) in 1995 saw substantial declines in suicide in young men.

\section{Aims}

To investigate whether areas with locally targeted suicide prevention activity implemented after 1995 experienced lower rates of young adult suicide, compared with areas without such activity.

\section{Method}

Localities with or without identified suicide prevention activity were compared during the period of the NYSPS implementation (1995-1998) and a period subsequent to implementation (1999-2002) to establish whether annual average suicide rates were lower and declined more quickly in areas with suicide prevention activity over the period 1995-2002.

\section{Results}

Male suicide rates were lower in areas with targeted suicide prevention activity (and higher levels of funding) compared with areas receiving no activity both during $(\mathrm{RR}=0.89,95 \% \mathrm{Cl}$ $0.80-0.99, P=0.030$ ) and after ( $R R=0.86,95 \% \mathrm{Cl} 0.77-0.96$, $P=0.009$ ) implementation, with rates declining faster in areas with targeted activity than in those without (13\% v. 10\% decline). However, these differences were reduced and were no longer statistically significant following adjustment for sociodemographic variables. There was no difference in female suicide rates between areas with or without targeted suicide prevention activity.

\section{Conclusions}

There was little discernible impact on suicide rates in areas receiving locally targeted suicide prevention activities in the period following the NYSPS.

\section{Declaration of interest}

None.
Young men (aged 20-34 years) in Australia experienced an epidemic rise in suicide between the 1970s and late 1990s. Rates more than doubled over this period, reaching a peak of approximately 40 per $100000 .^{1}$ In response, the Australian government implemented the first National Youth Suicide Prevention Strategy (NYSPS) in 1995-1997, ${ }^{2}$ which was subsequently widened in 1999 to focus on all age groups under the National Suicide Prevention Strategy (NSPS) and Living Is For Everyone (LIFE) framework. ${ }^{3}$ A range of prevention programmes and activities were implemented under the auspices of the initial NYSPS at national, state and local levels, including direct prevention approaches (e.g. primary mental healthcare, early and crisis interventions, and treatment, support and postvention initiatives) and system level approaches (e.g. education and training, communication, networking and intersectoral collaboration, and community development). ${ }^{4}$ In the period following the implementation of the NYSPS, young male suicide rates declined dramatically - differentially by method, ${ }^{1}$ socioeconomic status, ${ }^{5,6}$ and geographic area, ${ }^{5,6}$ and also after accounting for misclassification of suicide cases to other external causes. $^{7,8}$

It is not clear whether the NYSPS and associated state and local prevention activity implemented at the peak of the epidemic were effective in reducing suicide, or the extent to which any continued effects of other strategic responses might have been partly responsible for the subsequent decline in young male suicide rates. Previous evaluations of suicide prevention activity in Australia have been restricted to funded projects and programmes, and predominantly focused on process indicators (such as type and number of projects) rather than reductions in suicide. ${ }^{9,10}$ These evaluations noted that prevention activity was not focused especially on groups at risk (those with mental illness and previous history of suicidal behaviour) and on health service interventions relating to mental health and primary care services. ${ }^{10}$ Previous studies have investigated temporal associations between the onset of national suicide prevention activity and overall secular trends in suicide; ${ }^{1}$ however, no evaluation of suicide prevention activity has attempted to establish whether the intensity and scope of prevention measures in particular geographic areas and sociodemographic groups are associated with changes in rates of suicide.

This study aimed to evaluate the impact of the NYSPS on suicide rates in young adults by correlating locally targeted suicide prevention activity with corresponding area-based suicide rates, during the period of initial implementation (1995-1998) and subsequently when suicide rates in young men began to decline (1999-2002), to determine first whether suicide rates were lower in areas receiving targeted suicide prevention activity compared with areas not receiving such activity, and second whether suicide rates, particularly in young men, declined more quickly in areas receiving targeted suicide prevention activity than in areas that did not. Geographic markers of socioeconomic status and rurality were also used to determine whether differentials in suicide across social strata were also associated with the prevention programme at the peak of the youth suicide epidemic and in the subsequent period of decline. ${ }^{5,6,11-13}$

\section{Method}

Suicide data (ICD-9 codes E950-959 and ICD-10 codes X60$\mathrm{X} 84)^{14,15}$ were obtained from the Australian Bureau of Statistics for 1992-2002 and stratified by gender, 5-year age group (in those aged 20-34 years), country of birth (Australian-born, nonAustralian born) and statistical local area (median population 16548). Corresponding population counts were obtained from 
the 1996 and 2001 census populations. The suicide data used to correlate with suicide prevention activity relate to the 4-year period (1995-1998) spanning the 1996 census and the 4-year period (1999-2002) spanning the 2001 census. Suicide rates were related to when the original NYSPS was officially funded (19951997), ${ }^{2}$ and subsequent to its completion, to address any sustained effects. Suicide data for 1992-1994 (i.e. the previous 3 years) were also used to establish the suicide rate in a given area prior to the implementation of the NYSPS. A 'base rate' was incorporated into analyses to investigate whether any effects of suicide prevention activity were more prominent in areas of high or low suicide. Given substantial small-area boundary changes from year to year, ${ }^{5,6}$ it was necessary to code small-area suicide data to the corresponding statistical local area (SLA) in the 1996 census for the period 1995-1998 and to the 2001 census for the period 1999-2002. Death data where the cause of death was categorised as undetermined (E980-E989, Y10-Y34) were also included in sensitivity analyses, to assess any effects of possibly misclassified cases of suicide.

\section{Suicide prevention activity}

Information on suicide prevention activity was coded from a national stocktake of suicide prevention programmes and activities compiled by the Australian Institute of Family Studies. ${ }^{2,4}$ This first stocktake related to activity funded under the first NYSPS, plus activity funded by state and territory governments and non-government sources, and covered the period 19961997. ${ }^{2,4,16}$ A wide range of prevention programmes and activities were identified, with reported total funds of AUS\$76 million. The majority of prevention programmes related to community and professional education initiatives; crisis, early intervention, treatment and referral support; counselling and personal development initiatives; and health promotion initiatives. ${ }^{16}$ It is unclear what proportion of these programmes and activities were already established prior to 1995; however, a number of projects were continued and were part of the subsequent NSPS, established in $1999 .{ }^{9}$ For each prevention programme or activity described in the stocktake (910 entries) the following information was extracted: the target group, level of funding (where available), postcode and locality of the listed programme or organisation. Prevention programmes selected for inclusion were those for which it was clear that the identified programme or activity related to the immediate locale in which the organisation was based. Programmes or activities that targeted a broader region or the surrounding area of the locale in which the identified organisation was located, or where it was unclear as to which geographic area the programme related, were not included in primary analyses ( $49 \%$ of listed activities and $52 \%$ of total funds), but were included in a series of sensitivity analyses to investigate any diffuse effects of activity with a wider geographic focus, or the effects of potential misclassification of suicide prevention activity.

Programmes included regional or state level strategic initiatives. Postcodes and locality information were used to establish an SLA code for each listed prevention programme or activity. Post-office box addresses were reconciled to physical addresses where possible by a web search of the organisation. The resultant data-set was used to generate a summary variable of prevention programmes and activity for a given geographic area based on presence of suicide prevention activity and also on total level of funding for listed activities (in areas where programmes and activities could be accurately specified and where level of funding was reported).

As previous studies of Australian suicide have shown differentials in suicide by socioeconomic status, ${ }^{5,11}$ urban or rural residence, ${ }^{6,17}$ and country of birth, ${ }^{13,18,19}$ we considered these factors as potential confounding factors and as effect modifiers of any association between prevention activity and suicide. Area socioeconomic status was based on the economic resource index of the Socio-Economic Indexes for Areas (SEIFA) for 1996 and 2001. These SEIFA scores are aggregated, area-based measures of resources within local areas derived from census information and incorporate information on income and expenditure, home ownership rates, dwelling size and motor vehicle ownership. ${ }^{20}$ The economic resource index is a more internally consistent proxy measure of wealth than indices of education and occupation, and has also been associated with the greatest magnitude of socioeconomic differentials in suicide. ${ }^{11}$

Urban or rural residence was defined using the Rural, Remote and Metropolitan Area classification system, which combines population density (defined as 'personal distance' and calculated as an index of remoteness) and population size. ${ }^{21}$ Suicide rates were aggregated into two regions for this study: capital cities and metropolitan centres, and rural areas (comprising rural and remote centres, other rural areas and remote areas). Country of birth (Australian-born or not Australian-born) was also included in analyses, as previous studies have shown that migrant groups have suicide rates that can differ from those in the Australian-born population, ${ }^{19}$ and that migrant populations are differentially distributed by socioeconomic status and geographic area. ${ }^{13,22}$

Subgroup analysis of areas was also conducted based on previous area-based suicide rates, to investigate whether areas with previously higher suicide rates were more or less likely to receive targeted suicide prevention activity, and whether effects of targeted suicide prevention differed depending on whether the base rate of suicide was high or low. Gender-specific baseline suicide rates in areas receiving or not receiving targeted suicide prevention activity were stratified by population quintile derived from the previous period (1992-1994), such that the bottom quintile represented the areas with the lowest suicide rates and the top quintile represented the areas with the highest suicide rates. Areas were categorised according to suicide rates per 100000 in the ranges $0-3.1,3.2-5.9,6.0-13.8,13.9-20.2$ and more than 20.2 .

Data were stratified into each combination of statistical local area, gender, age group, country of birth group, urban-rural classification, socioeconomic status population quintile, baseline suicide rate quintile and presence or absence of suicide prevention activity, with corresponding counts of suicide, level of funding and population. This stratification resulted in 139 SLAs that had received locally targeted suicide prevention activity and 774 SLAs that had not. A further 71 SLAs were identified for inclusion in sensitivity analyses; these were areas where programmes or activities targeted a broader region or the surrounding area of the locale in which the identified organisation was located, or where it was unclear to which geographic area the programme related.

\section{Statistical analysis}

We compared areas with and without documented youth suicide prevention activity based on two periods: 1995-1998 and 19992002. Cross-sectional analyses were used to investigate whether areas with targeted suicide prevention activity had lower young adult suicide rates than areas without such activity in each period. Longitudinal analyses (across each period) were used to investigate whether declines in average annual suicide rates occurred faster in areas with targeted suicide prevention activity than in areas without. A series of variously adjusted negative binomial regression models of suicide counts (offset by the 
logarithm of the population) stratified by gender were specified to investigate whether the relative risk of suicide was lower in areas with locally targeted suicide prevention activity than in areas with no such activity for the periods 1995-1998 and 1999-2002. Negative binomial regression is a generalised Poisson model and incorporates a parameter for overdispersion where events have greater than Poisson variation. All models for 1995-1998 were adjusted for baseline (1992-1994) suicide rates. The secular change in suicide rates within areas with or without targeted suicide prevention activity was also investigated by comparing suicide rates across the two study periods. The extent of divergence or convergence between the slopes of suicide rates over this period was assessed by testing the interaction term between period (1995-1998 or 1999-2002) and type of area (receiving or not receiving targeted prevention activity).

Models for both 1995-1998 and 1999-2002 were adjusted for individual-level country of birth, area-based socioeconomic status (low-, middle- and high-status areas, representing 25\%, 50\% and $25 \%$ of the population respectively) and urban-rural residence (metropolitan, non-metropolitan). Effect modification was also investigated by migrant status, socioeconomic status and urbanrural residence, to investigate whether effects of suicide prevention activity differed within these strata. Subgroup analyses were also conducted by quintile of baseline suicide rate to investigate whether effects of suicide prevention activity differed in areas with previously higher or lower suicide rates. Sensitivity analyses were conducted by running the above series of models including areas where the geographic focus of the suicide prevention activity was unclear in addition to areas with locally targeted suicide prevention activity. Additional sensitivity analyses included cases where cause of death was classified as undetermined. Insufficient case numbers precluded meaningful analysis of the effects of specific types of prevention activity (e.g. whether population-based, or focusing on particular selected or high-risk subgroups).

Additional models restricted analyses to areas receiving locally targeted suicide prevention activity to investigate whether suicide rates were lower in areas reporting higher levels of funding. Funding categories were defined in thousands of AUS\$: 0-29, $30-250,>250$. Analyses of funding levels were conducted separately for each period (1995-1998 and 1999-2002) and also across each period (as described above) to investigate the rate of change in suicide rates within each level of funding. Analyses were carried out in Stata version 11.0 for Windows using 'nbreg'.

\section{Results}

Suicide rates in men aged 20-34 years were lower in areas with locally targeted suicide prevention activity than in areas without such prevention activity, in the periods before $(\mathrm{RR}=0.94,95 \%$ CI $0.83-1.06, P=0.337$ ), during $(\mathrm{RR}=0.89,95 \%$ CI $0.80-0.99$, $P=0.030)$ and after $(\mathrm{RR}=0.86,95 \%$ CI $0.77-0.96, P=0.009)$ the implementation of suicide prevention activity (Table 1). However, this difference in the period following 1995 was reduced and no longer statistically significant following adjustment for country of birth, socioeconomic status and urban-rural residence (Table 1). The absolute difference in suicide for men between these areas was approximately 2 suicides per 100000 (39 v. 37) for 1995-1998, and 1 per 100000 (35 v. 34) for 1999-2002, from adjusted models. In sensitivity analyses including both locally targeted suicide prevention activity and activity where the geographic focus was unclear, relative differences in suicide were smaller and not statistically significant for either period (1995-1998 and 1999-2002) (not shown). Including cases where cause of death was undetermined did not significantly affect relative differences in suicide.

Over the period 1999-2002 suicide rates declined significantly in areas both with and without locally targeted suicide prevention activity, declining faster in areas with such activity: $-13 \%$ ( $95 \%$ CI -23 to -1$)$ v. $-8 \%$ (95\% CI -16 to 1$)$, from adjusted models. However, there was no statistically significant difference in the rate of decline $(P=0.541$ for difference in percentage change, from the adjusted models) (Table 1).

Male suicide rates declined faster in non-Australian-born groups (compared with Australian-born groups) in areas that had targeted suicide prevention activity compared with those that did not $(-21 \%, 95 \%$ CI -42 to -7, v. $6 \%, 95 \%$ CI -16 to 34 , from adjusted models), but this difference in the rate of decline was not statistically significant $(P=0.153)$ (not shown). Male suicide rates declined faster in groups with higher ( $v$. lower) socioeconomic status, and in metropolitan ( $v$. rural) groups; however, there was no significant difference in the effects of suicide prevention activity by socioeconomic status or urban-rural residence (not shown). Male suicide rates declined faster in areas with previously high suicide rates (areas in the top two quintiles) than areas with lower suicide rates (not shown). There was no significant difference in the association between suicide prevention activity and suicide by previous level of suicide, although the difference in the rate of decline between areas receiving or not receiving locally targeted suicide prevention activity was greatest in the highest suicide rate group: $-17 \%$ ( $95 \%$ CI -32 to 1$)$ v. $1 \%$ (95\% CI -12 to 15$)$ from adjusted models $(P=0.109)$.

In areas receiving locally targeted prevention activity, male suicide rates were lower in areas with higher levels of funding for the period 1995-1998 after adjusting for baseline suicide rates $(P=0.001$ for trend). However, this trend was not statistically significant in the subsequent period (1999-2002) and the effect was attenuated further following adjustment for country of birth, socioeconomic status and urban-rural residence (Table 2; online Table DS1).

Suicide rates in women aged 20-34 years - substantially lower than male rates - were higher in areas with locally targeted prevention activity compared with areas receiving no prevention activity, but these differences were not statistically significant either during 1995-1998 ( $\mathrm{RR}=1.18,95 \%$ CI 0.94 to 1.49 , $P=0.149)$ or $1999-2002 \quad(\mathrm{RR}=1.09,95 \%$ CI 0.88 to 1.35 , $P=0.439)$. The absolute difference in suicide for women between these areas was approximately 1 suicide per 100000 for both periods (see adjusted rate estimates in Table 1), with higher rates in intervention areas. There was no significant difference in the effects of suicide prevention activity by socioeconomic status or urban-rural residence, by previous level of suicide, with inclusion of undetermined causes (not shown), or by level of prevention funding in areas receiving targeted suicide prevention activity (Table 2; online Table DS1).

\section{Discussion}

This study identified little, if any, effect on suicide rates in areas receiving locally targeted suicide prevention activities in young men over the life of the first National Youth Suicide Prevention Strategy (1995-1998) and in the subsequent period. Suicide rates in young men were already lower in areas with locally targeted suicide prevention activity and declined faster in the subsequent period in these areas. However, these differences were accounted for in analyses adjusting for socioeconomic status, urban-rural residence and migrant status. There was no significant difference 


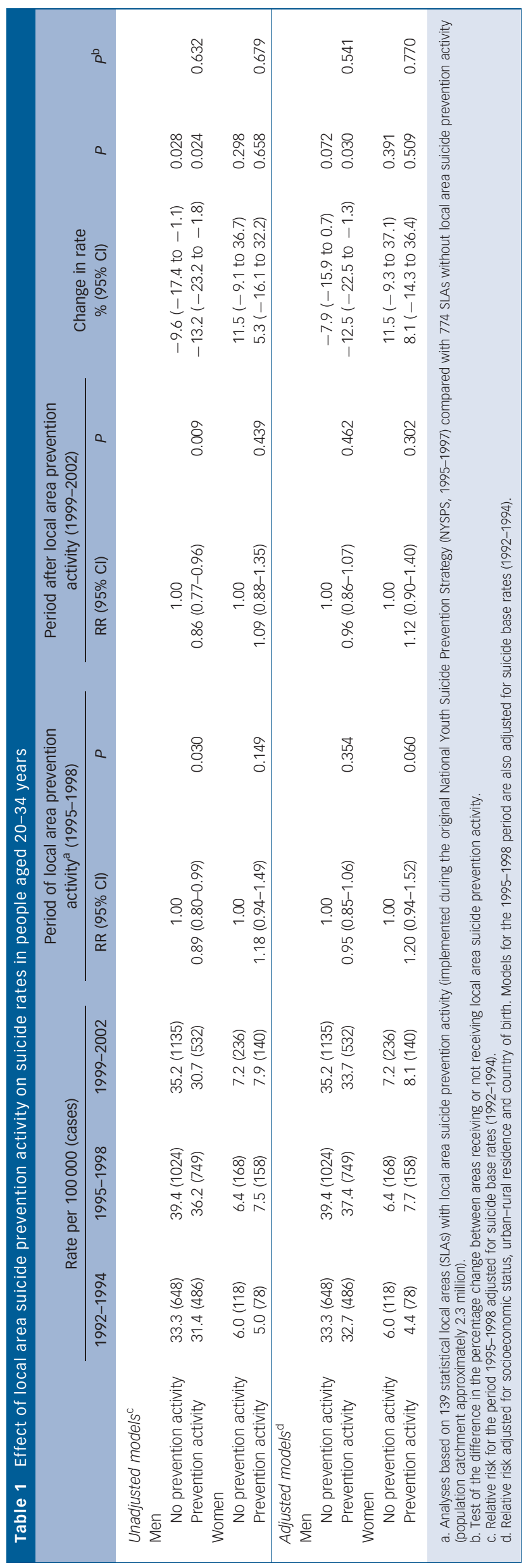

in suicide rates in young women between areas receiving or not receiving locally targeted suicide prevention activity. Effects of suicide prevention activity did not differ significantly across strata of migrant status, socioeconomic status, urban-rural residence or the previous level of suicide for both men and women.

\section{Limitations of the study}

The main limitation of our study relates to the enumeration and specification of prevention programmes and activities during the study period. We used publicly available reports of suicide prevention programmes and activities that were funded either by the federal government, state or territory governments or non-government sources identified as part of the NYSPS-funded national stocktake of suicide prevention activity covering 1996$1997 .^{2,4,16}$ This is likely to be an underenumeration of the total number of activities and programmes implemented during the study period, and excludes programmes not captured by the national stocktake, such as some non-government initiatives and initiatives that may have been implemented in the period after 1999 or funded under the subsequent national suicide prevention strategy. Additionally, there is likely to be bias in the way in which programmes were defined and operationalised for analysis. First, information about programmes was extracted from project descriptions contained in reports covering the initial phase of the national suicide prevention campaign. If programmes did not report funding amounts, or did not record sufficient information on the target areas for a given programme, they could not be included in the analysis. The final list of projects included in primary analyses comprised only programmes for which the target area could be specified, but any preventive effect might not have been limited to that particular area. It is also unclear what effect the population-level projects and interventions (such as web-based initiatives and telephone counselling) and other area-specific health service policies would have in areas classified in the analysis as being without any prevention activity. It is likely that the effect of this misclassification bias would be to reduce any observed associations between areas with or without prevention activity. However, the sensitivity analyses in this study, which included suicide prevention activities identified in the national stocktake with a broader (or unclear) geographic focus in addition to the locally targeted interventions, reduced differences between suicide rates in areas with or without suicide prevention activity.

Other important factors associated with proximal antecedents to suicide have not been included in this analysis. We did not include factors such as access to and availability of health services, which have been shown to account for geographic differences in Australian suicide rates between urban and rural areas in young men; ${ }^{13}$ nor were differentials in antidepressant consumption by socioeconomic status and geographic area, ${ }^{23}$ or sociodemographic differences in mental disorder prevalence, ${ }^{24}$ incorporated into analyses. It is likely that adjustment for these more proximate factors might further account for any modest associations observed between prevention activity and male suicide.

It is also important to note that measures of the actual reach or level of contact that individual organisations receiving prevention funding had with their target group were not used in our study. Rather, this is a fine-grained aggregate study of suicide and suicide prevention activity by local area and over time, and the 'reach' of suicide prevention was examined only with regard to funding. For instance, no information on suicide rates in the client bases of the various agencies providing social or mental health services to young people, before and after their receiving suicide prevention funding, was used in the analysis. Detailed studies of the individual organisations involved have yet to be conducted, 


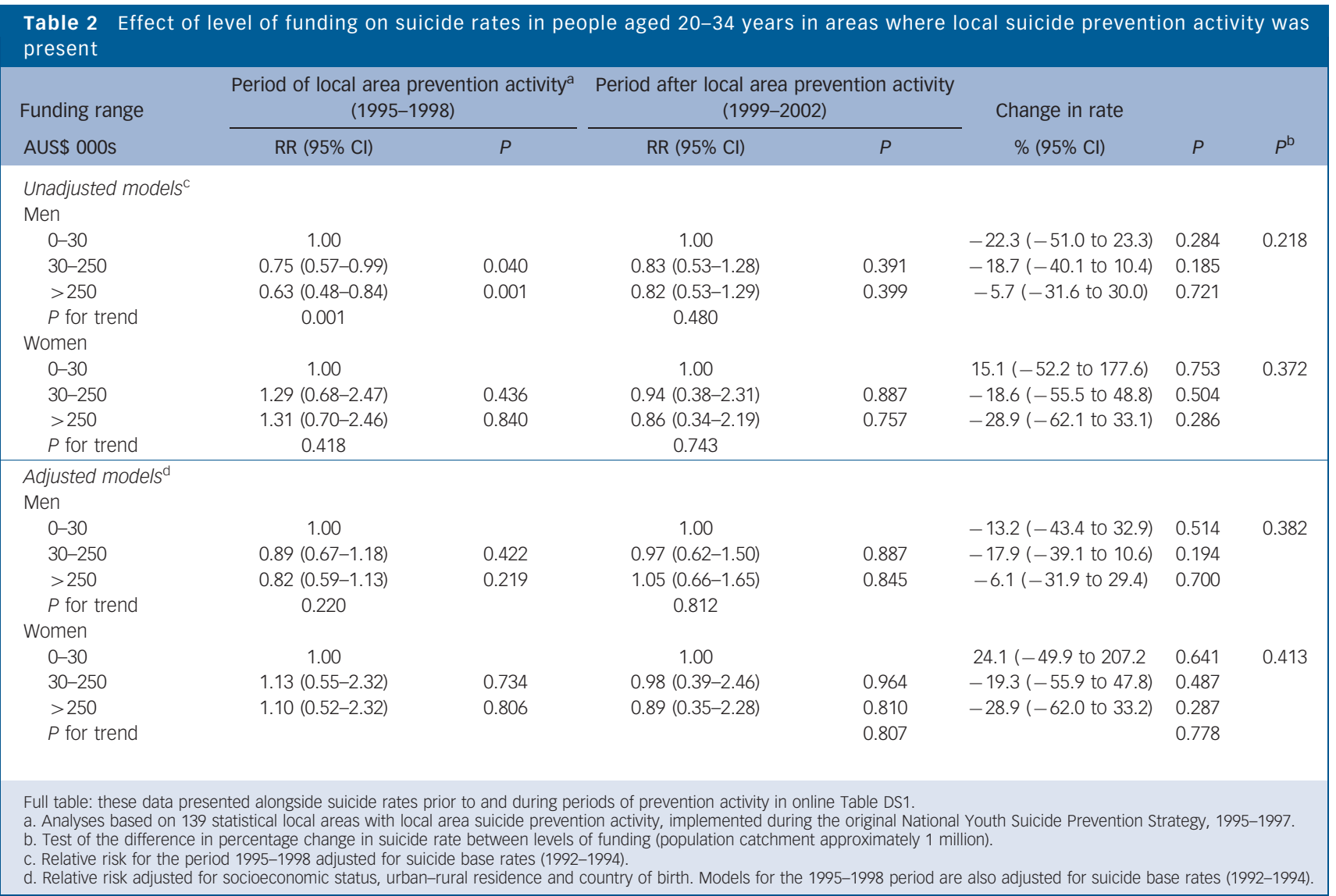

and it is possible that some of these organisations receiving suicide prevention funding did make a difference to suicide rates in the populations they dealt with directly.

An additional methodological consideration relates to the underenumeration of suicide cases due to the misclassification of likely suicide cases to undetermined and other external cause of death categories. ${ }^{7,25}$ Recent Australian studies have noted that suicides in the period after 2002 may be underenumerated by up to $16 \%,{ }^{7}$ compared with $5-10 \%$ in previous epochs, ${ }^{26,27}$ with an increase in likely suicide cases being classified under unintentional causes. ${ }^{7}$ However, these changes in the misclassification of cause of death relate to a more recent period (after 2002) than that covered by this study. Additionally, our sensitivity analyses incorporated causes of death with 'undetermined intent', which did not affect the reported findings.

\section{Comparison with other studies}

Lower suicide rates in young men in areas with prevention activity compared with areas without prevention activity may be consistent with suicide prevention projects having a beneficial effect on population suicide rates at the peak of the young male suicide epidemic, although in our study areas with locally targeted suicide prevention activity already had somewhat lower overall suicide rates compared with areas with no suicide prevention activity. Findings here differ from recent evaluations of similarly multidimensional suicide prevention strategies, which have suggested that such strategies may effect declines in suicidal behaviour if interventions are sufficiently well-defined, are based on pre-specified interventions, and are focused and sustained. ${ }^{28,29}$ Focused, non-randomised, community-based interventions in Germany have shown declines in population rates of attempted suicide (although not completed suicide). ${ }^{28}$ Also leadership and community involvement relating to the communication, dissemination and evaluation of mental health services and suicide assessment and prevention activity have been associated with sustained declines in suicide rates in the US Air Force. ${ }^{29}$ In contrast, interventions during the study period in the Australian context perhaps reflect a more disseminated facilitation of multidimensional policies and projects, and allocation of funds, across a range of contexts and population subgroups.

Previous evaluations of Australian suicide prevention initiatives, focused on detecting secular changes before and after the implementation of projects funded under the NYSPS, were unable to directly attribute the subsequent decline in young male suicide to the NYSPS and the subsequent NSPS. ${ }^{1}$ The lower suicide rates in men but not in women in areas with targeted prevention activity may reflect that the youth suicide epidemic was predominantly a male phenomenon. Even though the majority of suicide prevention projects focused on young people in general, ${ }^{4}$ the predominance of young male suicide was such that the main focus of implementation and uptake of projects in practice was on young men.

\section{Effect of socioeconomic status}

We had anticipated that there might have been effect measure modification of the association between prevention activity and suicide across socioeconomic strata and across strata of urbanrural residence, given the previously demonstrated socioeconomic status differentials in suicide in young men (higher suicide rates in areas of lower socioeconomic status), ${ }^{11}$ and divergence between high- and low-status groups, ${ }^{5}$ and between urban and rural areas, ${ }^{6,17}$ in the period subsequent to the implementation of the NYSPS. However, this was not the case. Previous studies of health promotion programmes implemented in other contexts have 
suggested that programmes are more likely to be implemented and supported by people in high-status groups, and that people in higher-status groups are more likely to respond to health promotion messages, for example being more likely than lowerstatus groups to engage in mammography screening, ${ }^{30,31}$ smoking cessation, ${ }^{32,33}$ and other examples of health promotion behaviour relating to diet and lifestyle factors associated with non-communicable diseases. ${ }^{34-36}$ Our study showed sharp declines in male suicide in higher-status groups and in metropolitan areas in general, but there was no difference in the effects of suicide prevention activity within strata of socioeconomic status or urban-rural residence.

\section{Implications}

We found little discernible impact of the distribution of funds to support suicide prevention activity on young adult suicide during the period of the initial implementation of the NYSPS (19951998 ) or in the subsequent period when male suicide rates started to decline (1999-2002). Although male suicide rates were lower and declined faster in areas receiving locally targeted suicide prevention activity (and higher levels of project funding) compared with areas receiving no such activity, these differences were small, pre-dated the funding and were attenuated in analyses adjusting for sociodemographic factors.

Potential alternative explanations for the decline in suicide in young men also require consideration, such as the role of primary care provision and emergence of the discourse and policy responses in primary mental healthcare, ${ }^{37,38}$ and increases in population antidepressant consumption over the 1990s. ${ }^{39}$ There also remains the role of gains in overall economic prosperity in Australia over the 1990s, especially in the context of the differential distribution of such gains across social strata, ${ }^{40}$ and diverging suicide rates between low- and high-socioeconomic-status groups. ${ }^{5}$ The study period also saw potentially important transitions in the characteristics of the labour force in young adults, through increased casualisation of employment, and the introduction of employment training programmes in the 1990s. ${ }^{1}$

The analytic approach employed in this study is a method that could be used for retrospective outcome evaluation of suicide prevention activity in the period subsequent to the implementation of the initial NYSPS, and clearer specification of suicide prevention activity by geographic area might provide more robust estimates of the effects of nationally funded suicide prevention activity, including the current LIFE framework. ${ }^{3}$ There has been a marked decline in young male suicide in Australia since the peak of the 'youth suicide epidemic' in 1997 which is not artefactual ${ }^{8}$ however, our study suggests that the initial implementation of youth suicide prevention activity had little impact on young adult suicide rates at the peak of the epidemic or on the subsequent decline in young adult suicide.

\footnotetext{
Andrew Page, PhD, Discipline of Epidemiology and Biostatistics, School of
Population Health, University of Queensland, Herston, Queensland, Australia; Richard Taylor, PhD, School of Public Health and Community Medicine, University of New South Wales, Randwick, New South Wales, Australia; David Gunnell, DSC, School of Social and Community Medicine, University of Bristol, Bristol, UK; Greg Carter, PhD, Centre for Brain and Mental Health Research, University of Newcastle, Callaghan, New South Wales, Australia; Stephen Morrell, PhD, School of Public Health and Community Medicine, University of New South Wales, Randwick, New South Wales, Community Medicine, University of New South Wales, Randwick, New South Wales,
Australia; Graham Martin, DPM, University of Queensland, and Royal Children's Australia; Graham Martin, DPM, University of Queensland, and Royal Children's
Hospital \& Brisbane North Child and Youth Mental Health Service, Centre for Psychiatry \& Clinical Neuroscience Research (Suicide Prevention Studies), Mental Health Centre, Herston, Queensland, Australia

Correspondence: Dr Andrew Page, Discipline of Epidemiology and Biostatistics, School of Population Health, University of Queensland, Herston Road, Herston, QLD 4006, Australia. Email: a.page@sph.uq.edu.au

First received 3 Mar 2011, final revision 23 May 2011, accepted 16 Jun 2011
}

\section{References}

1 Morrell S, Page AN, Taylor RJ. The decline in Australian young male suicide. Soc Sci Med 2007; 64: 747-54.

2 Australian Institute of Family Studies. Youth Suicide Prevention Programs and Activities - National Stocktake, October 1999. Australian Institute of Family Studies, 1999.

3 Department of Health and Ageing. Living is for Everyone (LIFE): A Framework for Prevention of Suicide in Australia. Commonwealth of Australia, 2008.

4 Mitchell P. Valuing Young Lives: Evaluation of the National Youth Suicide Prevention Strategy. Australian Institute of Family Studies, 2000

5 Page A, Morrell S, Taylor R, Carter G, Dudley M. Divergent trends in suicide by socio-economic status in Australia. Soc Psychiatry Psychiatr Epidemiol 2006; 41: 911-7.

6 Page A, Morrell S, Taylor R, Dudley M, Carter G. Further increases in rural suicide in young Australian adults: secular trends, 1979-2003. Soc Sci Med 2007; 65: 442-53.

7 Elnour A, Harrison J. Suicide decline in Australia: where did the cases go? Aust N Z J Public Health 2009; 33: 67-9.

8 Page A, Taylor R, Martin G. Recent declines in Australian male suicide are real, not artefactual. Aust N Z J Psychiatry 2010; 44: 358-63.

9 Headey A, Pirkis J, Merner B, VandenHeuvel A, Mitchell P, Robinson J, et al. A review of 156 local projects funded under Australia's National Suicide Prevention Strategy: overview and lessons learned. AeJAMH 2006; 5: 1-15.

10 Robinson J, McGorry P, Harris MG, Pirkis J, Burgess P, Hickie I, et al. Australia's National Suicide Prevention Strategy: the next chapter. Aust Health Rev 2006; 30: 271-6.

11 Page A, Morrell S, Taylor R. Suicide differentials in Australian males and females by various measures of socio-economic status, 1994-98. Aust N Z J Public Health 2002; 26: 318-24.

12 Taylor R, Page A, Morrell S, Harrison J, Carter G. Mental health and socio-economic variations in Australian suicide. Soc Sci Med 2005; 61: 1551-9.

13 Taylor R, Page A, Morrell S, Harrison J, Carter G. Social and psychiatric influences on urban-rural differentials in Australian suicide. Suicide Life Threat Behav 2005; 35: 277-90.

14 World Health Organization. International Statistical Classification of Diseases and Related Health Problems (ICD-9). WHO, 1978.

15 World Health Organization. International Statistical Classification of Diseases and Related Health Problems (ICD-10). WHO, 1992.

16 Mitchell P. A Content Analysis: First National Stocktake. Youth Suicide Prevention Bulletin 1999; 2: 2-7.

17 Dudley MJ, Kelk NJ, Florio TM, Howard JP, Waters BG. Suicide among young Australians, 1964-1993: an interstate comparison of metropolitan and rural trends. Med J Aust 1998; 169: 77-80.

18 Burvill PW, Woodings TL, Stenhouse NS, McCall MG. Suicide during 1961-70 migrants in Australia. Psychol Med 1982; 12: 295-308.

19 Taylor R, Morrell S, Slaytor E, Ford P. Suicide in urban New South Wales, Australia 1985-1994: socio-economic and migrant interactions. Soc Sci Med 1998; 47: 1677-86.

20 Australian Bureau of Statistics. 2001 Census of Population and Housing: Socio-Economic Indexes for Areas. Information Paper 2039.0. Commonwealth of Australia, 2003.

21 Department of Primary Industries and Energy, Department of Human Services and Health. Rural, Remote and Metropolitan Areas Classification: 1991 Census Edition. Australian Government Publishing Service, 1994.

22 Morrell S, Taylor R, Slaytor E, Ford P. Urban and rural suicide differentials in migrants and the Australian-born, New South Wales, Australia 1985-1994. Soc Sci Med 1999; 49: 81-91.

23 Page AN, Swannell S, Martin G, Hollingworth S, Hickie IB, Hall WD. Sociodemographic correlates of antidepressant utilisation in Australia. Med J Aust 2009; 190: 479-83.

24 Taylor R, Page A, Morrell S, Carter G, Harrison J. Socio-economic differentials in mental disorders and suicide attempts in Australia. Br J Psychiatry 2004; 185: 486-93.

25 De Leo D. Suicide mortality data need revision. Med J Aust 2007; 186: 157-8.

26 Burvill PW, McCall MG, Stenhouse NS, Woodings TL. The relationship between suicide, undetermined deaths and accidental deaths in the Australian born and migrants in Australia. Aust N Z J Psychiatry 1982; 16: $179-84$

27 Cantor $\mathrm{CH}$, Dunne MP. Australian suicide data and the use of 'undetermined' death category (1968-1985). Aust N Z J Psychiatry 1990; 24: 381-4. 
28 Hegerl U, Mergl R, Havers I, Schmidtke A, Lehfeld H, Niklewski G, et al. Sustainable effects on suicidality were found for the Nuremberg alliance against depression. Eur Arch Psychiatry Clin Neurosci 2010; 260: 401-6.

29 Knox KL, Pflanz S, Talcott GW, Campise RL, Lavigne JE, Bajorska A, et al. The US Air Force suicide prevention program: implications for public health policy. Am J Public Health 2010; 100: 2457-63.

30 Puddu M, Demarest S, Tafforeau J. Does a national screening programme reduce socioeconomic inequalities in mammography use? Int J Public Health 2009; 54: 61-8

31 Taylor R, Ivanov O, Page A, Brotherton J, Achat H, Close G. Predictors of non-attendance from BreastScreen NSW in women who report current mammography screening. Aust N Z J Public Health 2003; 27: 581-7.

32 Niederdeppe J, Fiore MC, Baker TB, Smith SS. Smoking-cessation media campaigns and their effectiveness among socioeconomically advantaged and disadvantaged populations. Am J Public Health 2008; 98: 916-24.

33 Sorensen G, Barbeau E, Hunt MK, Emmons K. Reducing social disparities in tobacco use: a social-contextual model for reducing tobacco use among blue-collar workers. Am J Public Health 2004; 94: 230-9.

34 Goldman RE, Barbeau E, Hunt MK, Acevedo-Garcia D, Emmons KM, Gagne J, et al. Perceptions of health promotion and cancer prevention among adults in working-class occupations and neighborhoods. Health Educ Behav 2008; 35: $777-90$.

35 Turrell G, Hewitt B, Patterson C, Oldenburg B, Gould T. Socioeconomic differences in food purchasing behaviour and suggested implications for diet-related health promotion. J Hum Nutr Diet 2002; 15: 355-64.

36 Young CM, Batch BC, Svetkey LP. Effect of socioeconomic status on food availability and cost of the Dietary Approaches to Stop Hypertension (DASH) dietary pattern. J Clin Hypertens (Greenwich) 2008; 10: 603-11.

37 Harris MG, Burgess PM, Pirkis JE, Slade TN, Whiteford HA. Policy initiative to improve access to psychological services for people with affective and anxiety disorders: population-level analysis. Br J Psychiatry 2011; 198 99-108.

38 Hickie I, Groom G. Primary care-led mental health service reform: an outline of the Better Outcomes in Mental Health Care initiative. Australas Psychiatry 2002; 10: 376-82.

39 Mant A, Rendle VA, Hall WD, Mitchell PB, Montgomery WS, McManus PR, et al. Making new choices about antidepressants in Australia: the long view 1975-2002. Med J Aust 2004; 181 (suppl 7): S21-4.

40 Saunders P. Examining Recent Changes in Income Distribution in Australia. SPRC Discussion Paper 130. Social Policy Research Centre, UNSW, 2003.

\section{0 Psychiatric exceptionalism

The invocation of exceptionalism can be beneficial, but it can also exact a cost. Employed nationalistically - for example, 'American exceptionalism' - it can inspire the citizenry or rally a mob. 'HIV exceptionalism' protects vulnerable patients but may compromise public health. Whenever psychiatry asserts special status relative to other medical disciplines, 'psychiatric exceptionalism' is implied. Supplemental confidentiality protections, the care of 'clients' rather than patients, the psychopharmacologist designation, and other practices emphasise the other-ness of psychiatry. Cumulatively, these attempts to be or to appear to be 'exceptional' have the unintended effect of reinforcing the image of psychiatrists and their patients as exceptions. 